\title{
Microscopic testicular sperm extraction or post-operative sperm reversal in functional Leydig cell tumor: case report
}

\author{
Zhongyan Liang ${ }^{1}$, Zhanwang $\mathrm{He}^{2}$, Chong Chen ${ }^{3}$, Fengbin Zhang $^{1}$, Jingping Li $^{1}$, Jinggen Wu ${ }^{1}$ \\ ${ }^{1}$ Reproductive Medicine Center, Department of Urology and Andrology, Women's Hospital, School of Medicine, Zhejiang University, Hangzhou \\ 310006, China; ${ }^{2}$ Department of Reproductive Medicine Center, Ninghai Maternal and Child health Hospital, Ninghai 315600, China; ${ }^{3}$ Department \\ of Ultrasonography, Women's Hospital, School of Medicine, Zhejiang University, Hangzhou 310006, China \\ Correspondence to: Jinggen Wu. Reproductive Medicine Center, Department of Urology and Andrology, Women's Hospital, School of Medicine, \\ Zhejiang University, Hangzhou 310006, China. Email: wujinggen@zju.edu.cn.
}

\begin{abstract}
Leydig cell tumors are rare testicular tumors in adults. Hormonal activity is found in $20 \%$ of the cases with endocrine abnormalities, which may result in azoospermia. The appropriate management to achieve oncologic control and simultaneously obtain sperm remains a challenge. A patient sought assistance with fertility after a diagnosis of azoospermia accompanied by unilateral suspected Leydig cell tumor. The patient underwent unilateral orchidectomy along with microscopic testicular sperm extraction (mTESE) for sperm identification. Rare teratospermia was found during mTESE, hormones tended to be normal, and sperm reversal appeared postoperatively. Postoperative semen examination revealed oligozoospermia, and the parameters decreased further after 3 months. The androgen rebound effect promoted sperm level over the baseline within 3 months after surgery. The anticipated sperm reversal postoperatively rather than mTESE during routine orchidectomy achieved sperm acquisition. This case particularly highlights the androgen rebound effect, which elevated the sperm level beyond baseline within 3 months after surgery. Sperm cryopreservation is thus strongly recommended 3 months postoperatively and no more than 4 months.
\end{abstract}

Keywords: Leydig cell tumor; microdissection testicular sperm extraction (mTESE); infertility; azoospermia

Submitted Mar 19, 2019. Accepted for publication Aug 02, 2019.

doi: 10.21037/tau.2019.08.10

View this article at: http://dx.doi.org/10.21037/tau.2019.08.10

\section{Introduction}

Approximately $5 \%$ to $6 \%$ of all testis tumors are non-germ cell tumors (1). Leydig cell tumors account for $1 \%$ to $3 \%$ of all testicular tumors in adults (2), while hormonal activity is found in $20 \%$ of the cases with endocrine abnormalities. However, azoospermia, reported to be a symptom of this tumor, is only mentioned in a few reports $(3,4)$. Doctors usually perform orchidectomy or testis-sparing surgery to achieve oncologic control. With the development of the microsurgical technique, we focus on a sperm-obtaining strategy by microdissection testicular sperm extraction (mTESE) during routine orchidectomy. However, the case we present was found to have sperm reversal and androgen rebound effect after surgery.

\section{Case presentation}

In 2018, our center implemented orchidectomy in a case of azoospermia accompanied by unilateral suspected Leydig cell tumor (LCT), and clinical data were collected. In this case report, we retrospectively evaluated the efficacy of the surgery and the prognosis of the case and compared it with conventional and novel methods in the literature. This case study was carried out in the Department of Urology and Andrology, Women's Hospital, School of Medicine, Zhejiang University. In accordance with the Women's Hospital, Zhejiang University Institutional Review Board (IRB) policies, case reports are not considered to be a research subject to IRB review and are thus exempt from formal IRB approval for publication. However, we obtained 
Table 1 Sexual hormones and tumor markers and semen parameters at baseline, after surgery, and in the follow-up

\begin{tabular}{|c|c|c|c|c|c|c|c|c|c|c|c|}
\hline Time & \multicolumn{4}{|c|}{ Sexual hormones } & \multicolumn{3}{|c|}{ Tumor markers } & \multicolumn{4}{|c|}{ Semen parameters } \\
\hline 3 months after surgery & 4.8 & 5.0 & 8.9 & 63.5 & - & - & - & 2.2 & 5.0 & 11 & 26.0 \\
\hline
\end{tabular}

$\Pi$, total testosterone; AFP, alpha fetoprotein; CEA, carcinoembryonic antigen; AST, aspartate transaminase; ALT, alanine transaminase; Vol, volume; Con, concentration; TCN, total sperm count; PR, progressive rate.

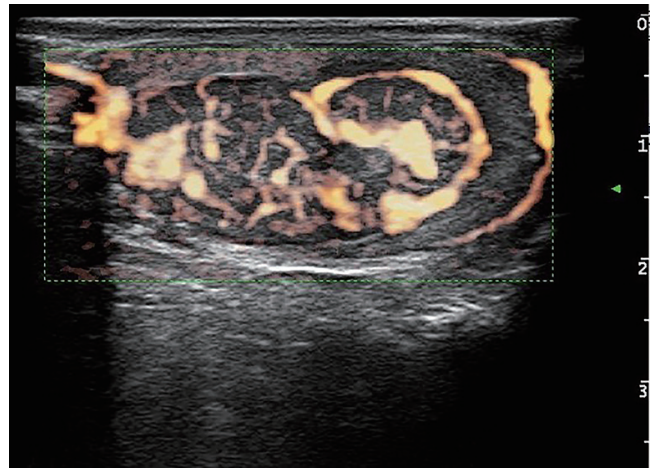

Figure 1 Ultrasound of a left Leydig cell tumor with XFLOW maps. The tumor appears as a hypoechoic non-homogeneous mass with high vascularity in parenchymal and peripheral tissue.

written consent from the patient for the publication of our findings. Here, through a description of the case and review of the relevant literature, we offer our thoughts on the strategy of obtaining sperm for fertility specialists.

A 28-year-old male presented to our andrology department with a 6-month history of primary infertility. The female partner was referred to our infertility clinic and her initial investigations were unremarkable. The couple was having sexual intercourse two to three times a week with normal ejaculation. He did not report any sexual dysfunction and hypaphrodisia in the past, nor any gynecomastia or breast tenderness. He had never fathered children and never underwent any previous surgical operation of the genitalia. Semen analysis in another hospital revealed azoospermia after centrifugation. Physical examination of the male revealed normal secondary sexual characteristics. The left testis was approximately $15 \mathrm{~mL}^{3}$ in size as measured by palpation and confirmed with the use of a Prader orchidometer, and the consistency was firm; however, no testicular masses were palpable. The right testicle was of normal consistency with a volume of approximately $6 \mathrm{~mL}^{3}$. Repeated semen analysis in our laboratory reconfirmed azoospermia, and the neutral $\alpha$-glucosidase of 23.8 MU (ref. $>20 \mathrm{MU} / \mathrm{per}$ ejaculation) and seminal fructose of $25 \mu \mathrm{mol}$ (ref. $>13 \mu \mathrm{mol} /$ per ejaculation) were of the normal level. Laboratory investigations of hormones were as follows: total T $29.3 \mathrm{nmol} / \mathrm{L}$ (ref. $6.6-35.0 \mathrm{nmol} / \mathrm{L}$ ), $\mathrm{LH}<0.1 \mathrm{IU} / \mathrm{L}$ (ref. 2.2-8.4 IU/L), FSH <0.1 IU/L (ref. 2.3-9.5 IU/L), $\mathrm{E}_{2}$ $170.0 \mathrm{pmol} / \mathrm{L}$ (ref. $28.0-156.0 \mathrm{pmol} / \mathrm{L}$ ), and PRL $11.7 \mathrm{ng} / \mathrm{mL}$ (ref. $6.1-17.0 \mathrm{ng} / \mathrm{mL}$ ) (Table 1).

A testicular ultrasound scan illustrated a well-defined hypoechoic mass near the center of the left testis. It measured $31 \times 19 \times 15 \mathrm{~mm}^{3}$ and contained massive blood flow signals by XFLOW maps (Figure 1). A decreased sized of the right testis and bilateral normal epididymis were noted. Further laboratory investigations revealed the following: HCG $<0.1 \mathrm{IU} / \mathrm{L}$ (ref. $<5.3 \mathrm{IU} / \mathrm{L}$ for male adult), alpha-fetoprotein (AFP) $2.6 \mathrm{ng} / \mathrm{mL}$ (ref. $<7.0 \mathrm{ng} / \mathrm{mL}$ ), and carcinoembryonic antigen (CEA) $1.9 \mathrm{ng} / \mathrm{mL}$ (ref. $<5.0 \mathrm{ng} / \mathrm{mL}$ ). He then underwent peripheral leukocyte karyotyping which revealed a 46, XY genotype and no microdeletion of azoospermia factor (AZF) on the $\mathrm{Y}$ chromosome.

Based on the investigations as mentioned above, the most likely diagnosis was a functional gonadal stromal tumor which secreted testosterone and estradiol. After treatment options were discussed, the patient elected to pursue left orchidectomy and mTESE to identify and cryopreserve sperm if possible. Considering that a large amount of radiation from computed tomography (CT) would affect the spermatozoon, chest X-ray film and ultrasound of the abdomen instead of CT was applied, and no obvious evidence of metastatic disease was found. The other preoperatively examinations revealed a significantly 


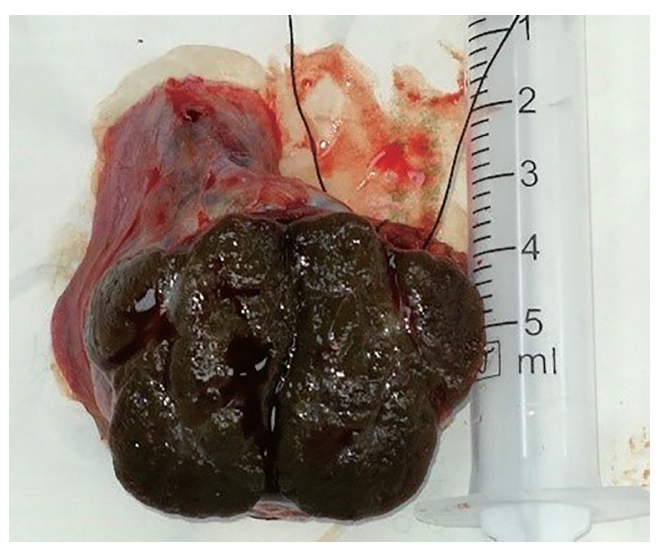

Figure 2 Leydig cell tumors of the left testis.

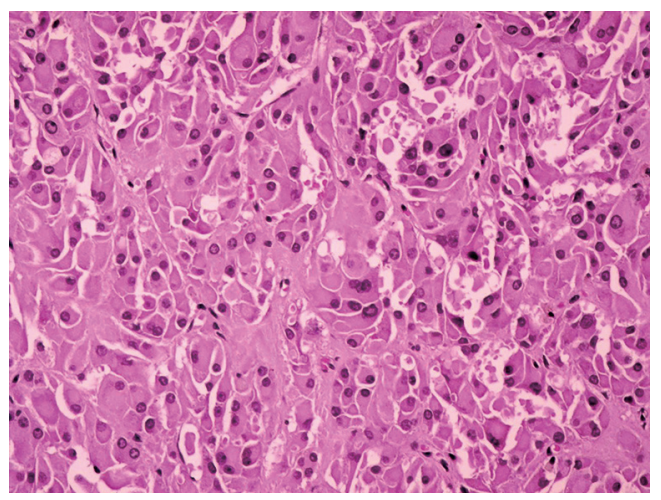

Figure 3 The Leydig cell tumor was composed of polygonal cells predominantly with eosinophilic cytoplasm; hematoxylin-eosin stain $\times 40$.

elevated level of hemoglobin $171 \mathrm{~g} / \mathrm{L}$ (ref. 120-160 g/L), aspartate transaminase (AST) $60 \mathrm{U} / \mathrm{L}$ (ref. 15-40 U/L), and alanine transaminase (ALT) $131 \mathrm{U} / \mathrm{L}$ (ref. 9-50 U/L). The left orchidectomy and mTESE under local anesthesia were then performed. The operation was performed one month after the initial visit to the hospital. There was a firm palpable lesion restricted in the tunica and not involved with the epididymis. On section, a single, firm, dark brown nodule was located in the center, with approximately normal testicular tissue surrounding it (Figure 2). The mTESE procedure was performed on the peripheral tissue under the surgical microscope (Carl Zeiss, S88, Germany) with a magnification of $10 \times$ to $15 \times$. Some seminiferous tubules were collected and examined under the microscope (Leica $400 x$ ) to analyze the presence of sperm. Rare sperm with the predominant deformity of the sperm head was observed

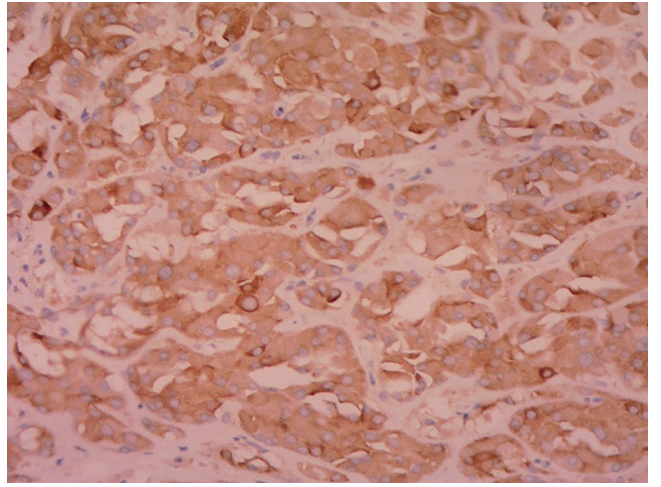

Figure 4 The immunohistochemical staining results of this Leydig cell tumors showed diffuse positive staining for inhibin; $\times 20$.

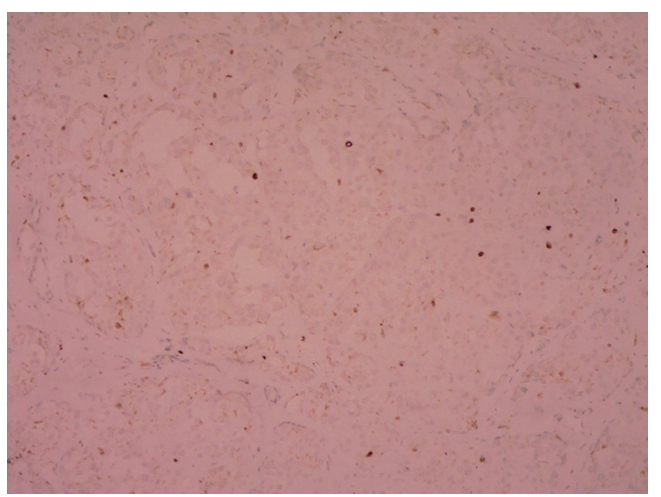

Figure 5 The immunohistochemical staining results of this Leydig cell tumors showed negative staining for Ki-67 (about $2 \%+$ ); $\times 10$.

indicating insufficiency and impaired spermatogenesis; cryopreservation of sperm was thus not recommended.

Postoperative pathological findings confirmed a left LCT $3 \times 2 \times 1 \mathrm{~cm}^{3}$ in size. The tumor was composed of polygonal cells predominantly with eosinophilic cytoplasm. There was almost no nuclear division and the mitotic count was $<1 / 10$ hpf (Figure 3). No capsular or vascular invasion was seen. The immunohistochemical profile showed diffuse positive staining for inhibin (Figure 4), calretinin, vimentin, melan-A, and SF1, and negative staining for cytokeratin and Ki-67 (about 2\%+) (Figure 5). After three months, the physical examination of the inguen and ultrasound of the scrotum showed no enlarged lymph nodes. The patient was evaluated five months postoperatively. The blood tests, including hemoglobin $148 \mathrm{~g} / \mathrm{L}$, AST32 U/L, ALT33 U/L, and the tumor markers AFP and $\beta$-hCG were still negative. 


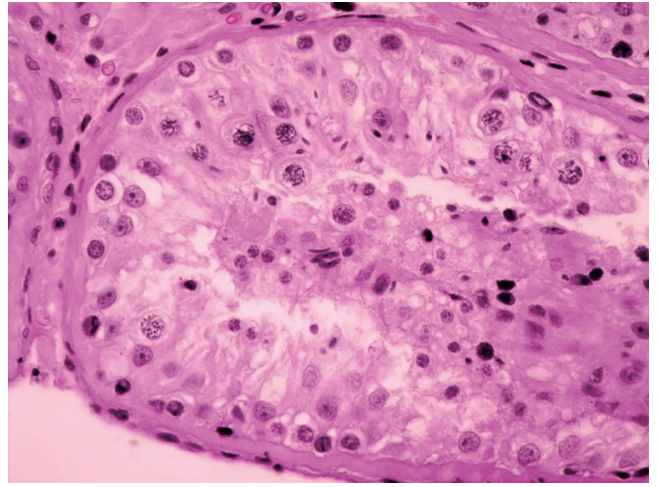

Figure 6 In the macroscopically normal area (non-neoplastic testicular tissue), the seminiferous tubules showed Sertoli cells with complete spermatogenesis in individual seminiferous tubules; hematoxylin-eosin stain $\times 40$.

Abdominal and pelvis contrast-enhanced CT and chest CT scan were applied, and no obvious evidence of metastatic disease was discovered. The macroscopically normal area showed hypospermatogenesis with primary or secondary spermatocytes, and spermatogenesis with spermatid or sperm could be found in individual seminiferous tubules (Johnsen score $=7$ ) (Figure 6). Microcalcification could also be found in individual tubules. Three months after the left orchidectomy, a repeated semen analysis indicated sperm reversal with a total count of $11 \times 10^{6}$ (ref. $\geq 39 \times 10^{6}$ ), a concentration of $5.0 \times 10^{6} / \mathrm{mL}$ (ref. $\geq 15 \times 10^{6} / \mathrm{mL}$ ), and a progressive rate of $26 \%$ (ref. $\geq 32 \%$ ). Laboratory investigations revealed a normal level of sexual hormones: serum LH $4.8 \mathrm{U} / \mathrm{L}, \mathrm{FSH} 5.0 \mathrm{U} / \mathrm{L}$, testosterone $8.9 \mathrm{nmol} / \mathrm{L}$, and $\mathrm{E}_{2} 63.5 \mathrm{pmol} / \mathrm{L}$. Semen analysis showed significantly decreased parameters, including a total sperm count of $5.2 \times 10^{6}$ and a sperm concentration of $2.0 \times 10^{6} / \mathrm{mL}$ five months postoperatively with the hormonal investigations being normal. He returned to our andrology department after one month with a repeated semen analysis, which indicated oligospermia of $2.0 \times 10^{6} / \mathrm{mL}$ in concentration and $4.0 \times 10^{6}$ in the total count. Intracytoplasmic sperm injection (ICSI) was suggested, but the patient refused at that time.

\section{Discussion}

Leydig cell tumors are rare neoplasms originating from the gonadal stroma and account for $1 \%$ to $3 \%$ of all testicular tumors in adults and $4 \%$ in prepubertal children $(2,5)$. They are most commonly unilateral, and $3 \%$ to $9 \%$ have been estimated to be bilateral (6). The presenting features are enlarged testis, gynecomastia, and sexual dysfunction. Testicular sonography is sufficiently sensitive to detect asymptomatic tumors incidentally. In this case, the testicular mass was not touched, but the shape of the testicle increased and it changed to a firm consistency. Ultrasound images could more clearly show the nature of the mass (7).

Hormonal activity is found in $20 \%$ of the cases with endocrine abnormalities, which may lead to gynaecomastia or loss of libido. However, azoospermia is not usually reported as a symptom of this tumor $(3,4)$. The most commonly produced hormones are testosterone and estrogen. The serum gonadotropin levels are usually low due to the negative feedback of the hypothalamuspituitary-gonads axis (8). This case involved a testosteronesecreting tumor and azoospermia. The mechanism of azoospermia is likely to be autonomous production of testosterone by the abnormal left testis tumor leading to suppression of gonadotropins. Impaired spermatogenesis in both the left uninvaded normal tissue and the right testis occurred, and moreover, rare sperm was observed in the mTESE procedure, and a very patchy spermatic stage macroscopically (Johnsen score $=7$ ) confirmed hypospermatogenesis. The fact that tumor removal resulted in the reversal of spermatogenesis, in this case, indicates the feedback inhibition characteristics of the gonad axis caused by the tumor before surgery.

Inguinal orchidectomy is the initial treatment in order to remove the primary tumor and to establish the diagnosis. Testis sparing surgery, a more conservative therapy, suggested by some authors, has been proven feasible and safe and could be regarded as first-line therapy in cases of benign, small tumors (under $25 \mathrm{~mm}$ ), and young men $(9,10)$. However, in our case, the tumor was consisted of $1 / 3$ of the left testis in volume and was located in the center; meanwhile, no tumor was found in the contralateral testis. We preferred the inguinal orchidectomy to testis-sparing surgery mainly because of the undefined characteristics of the tumor, and furthermore, the intraoperative frozen section diagnosis was not needed. Considering the undefined characteristics of the tumor and possible subsequent chemotherapy or radiotherapy, we tried to apply the mTESE in the para-tumor testicular tissue while performing orchidectomy (11). Therefore, high-dose CT was replaced by chest $\mathrm{X}$-ray and an abdominal ultrasound to exclude metastatic signs initially. Local anesthesia instead of general anesthesia was performed to avoid affecting sperm. With the magnification of $10 \times$ to $12 \times$, we could define the border of the tumor and explore some tubules 
outside. The examination of tubules indicated rare and abnormal spermatozoon. Neither ICSI nor sperm freezing was suggested. Malignant testicular tumors (either germ cell or non-germ cell) demonstrated markedly impaired spermatogenesis throughout the testis, being particularly impaired in areas adjacent to the tumor $(12,13)$. However, in this case, the elevated testosterone and $\mathrm{E}_{2}$ elicited the negative feedback action on $\mathrm{LH}$ and FSH secretion, which primarily caused the suppression of spermatogenesis (8).

After the removal of the tumor, hormone levels returned to normal, and sperm reversal appeared, which confirmed the assumption mentioned above. The hormones sustained the normal level in the six following months; however, the count (concentration \& total sperm counts) of sperm with ejaculation decreased markedly after three months and then remained at the same level. This interesting phenomenon is reminiscent of the androgen rebound effect. These excessive endogenous androgens suppressed gonadotrophin secretion and spermatogenesis. After left orchidectomy of the tumor, androgen secretion decreased, and there could have been a surge of FSH and LH, with spermatogenesis recommencing. However, the androgen rebound effect cannot be maintained for more than four months because of the spermatogenic cycle and the transient secretion surge of sex hormones $(14,15)$. Sperm cryopreservation is strongly recommended at three months postoperatively and no more than four months. We regret that sperm-freezing was not applied three months after surgery. Considering the sever oligospermia for the residual testis, ICSI with sperm in ejaculation is suggested. One year after surgery, ICSI with sperm from ejaculation was applied. Ultimately, 5 of $7 \mathrm{MII}$ oocytes were fertilized normally to form four early division embryos and cryopreserved for subsequent transplantation.

Leydig cell tumors are mostly benign, but $10 \%$ of them have the potential of metastasizing. In our case, preoperative chest film and abdominal ultrasound did not indicate any metastasis signs, nor did postoperative CT scan of the chest and abdomen. The size and mitotic activity of the original tumor appear to be the best predictors of outcome $(5,16)$. Our case presented benign histopathological features such as small size, lower mitotic count, less nuclear pleomorphism, and no capsular and vascular invasion. Furthermore, the results of immunoreactive tests were consistent with the existing literature $[\mathrm{SF} 1(+)$, calretinin $(+)$, inhibin $(+)$, vimentin (+), CK (-), melan-A(+), Ki-67 (approximately $2 \%+)](17-19)$, which provides complementary information to our pathology reports. Nevertheless, regular follow-up is recommended.

\section{Conclusions}

Functional testicular Leydig cell tumors can affect spermatogenic function by secreting excessive hormones of testosterone and $\mathrm{E}_{2}$. After surgical resection of the tumor, hormones tend to be normal and sperm reversal appears. Moreover, the androgen rebound effect, which leads to the elevated level of sperm beyond the baseline, can occur 3 months after surgery. Therefore sperm cryopreservation is recommended. For patients involved in bilateral orchidectomy or postoperative radiotherapy/chemotherapy, simultaneous intraoperative mTESE in paraneoplastic tissue, and cryopreservation of normal spermatozoon are suggested (11). Furthermore, preimplantation genetic screening (PGS) with the thawed sperm can be applied to exclude aneuploid karyotype embryos (20).

\section{Acknowledgments}

The authors would like to thank Dr. Haiyan Shi for her contribution in providing immunocytochemistry data and pathologic diagnosis.

\section{Footnote}

Conflicts of Interest: The authors have no conflicts of interest to declare.

Ethical Statement: The authors are accountable for all aspects of the work in ensuring that questions related to the accuracy or integrity of any part of the work are appropriately investigated and resolved. Written informed consent was obtained from the patient for publication of this case report and any accompanying images.

\section{References}

1. Dilworth JP, Farrow GM, Oesterling JE. Non-germ cell tumors of testis. Urology 1991;37:399-417.

2. Emerson RE, Ulbright TM. Morphological approach to tumours of the testis and paratestis. J Clin Pathol 2007;60:866-80.

3. Fallick ML, Lin WW, Lipshultz LI. Leydig cell tumors presenting as azoospermia. J Urol 1999;161:1571-2.

4. Markou A, Vale J, Vadgama B, et al. Testicular leydig cell tumor presenting as primary infertility. Hormones (Athens) 2002;1:251-4.

5. Cheville JC. Classification and pathology of testicular 
germ cell and sex cord-stromal tumors. Urol Clin North Am 1999;26:595-609.

6. Mostofi FK PE. Tumors of the male genital system. Atlas of Tumor Pathology. 8 ed. Washington, DC: Armed Forces Institute of Pathology; 1973:86-99.

7. Leonhartsberger N, Ramoner R, Aigner F, et al. Increased incidence of Leydig cell tumours of the testis in the era of improved imaging techniques. BJU Int 2011;108:1603-7.

8. Reznik Y, Rieu M, Kuhn JM, et al. Luteinizing hormone regulation by sex steroids in men with germinal and Leydig cell tumours. Clin Endocrinol (Oxf) 1993;38:487-93.

9. Chaabouni A, Chabchoub K, Rebai N, et al. Testis sparing surgery for Leydig cell tumors: New three cases and review of the current literature. Afr J Urol 2013;19:66-8.

10. Loeser A, Vergho DC, Katzenberger T, et al. Testissparing surgery versus radical orchiectomy in patients with Leydig cell tumors. Urology 2009;74:370-2.

11. Furuhashi K, Ishikawa T, Hashimoto H, et al. Oncotesticular sperm extraction: testicular sperm extraction in azoospermic and very severely oligozoospermic cancer patients. Andrologia 2013;45:107-10.

12. Ho GT, Gardner H, DeWolf WC, et al. Influence of testicular carcinoma on ipsilateral spermatogenesis. J Urol 1992;148:821-5.

13. Ho GT, Gardner H, Mostofi K, et al. The effect of testicular nongerm cell tumors on local spermatogenesis. Fertil Steril 1994;62:162-6.

Cite this article as: Liang Z, He Z, Chen C, Zhang F, Li $\mathrm{J}, \mathrm{Wu} \mathrm{J}$. Microscopic testicular sperm extraction or postoperative sperm reversal in functional Leydig cell tumor: case report. Transl Androl Urol 2019;8(5):556-561. doi: 10.21037/ tau.2019.08.10
14. Vandekerckhove P, Lilford R, Vail A, et al. Androgens versus placebo or no treatment for idiopathic oligo/ asthenospermia. Cochrane Database Syst Rev 2000;(2):CD000150.

15. Wang C, Chan CW, Wong KK, et al. Comparison of the effectiveness of placebo, clomiphene citrate, mesterolone, pentoxifylline, and testosterone rebound therapy for the treatment of idiopathic oligospermia. Fertil Steril 1983;40:358-65.

16. Middendorff R, Davidoff MS, Mayer B, et al. Neuroendocrine characteristics of human Leydig cell tumours. Andrologia 1995;27:351-5.

17. Bremmer F, Schweyer S, Martin-Ortega M, et al. Switch of cadherin expression as a diagnostic tool for Leydig cell tumours. Apmis 2013;121:976-81.

18. Iczkowski KA, Butler SL. New immunohistochemical markers in testicular tumors. Anal Quant Cytol Histol 2006;28:181-7.

19. McCluggage WG, Shanks JH, Arthur K, et al. Cellular proliferation and nuclear ploidy assessments augment established prognostic factors in predicting malignancy in testicular Leydig cell tumours. Histopathology 1998;33:361-8.

20. Spermon JR, Ramos L, Wetzels AM, et al. Sperm integrity pre- and post-chemotherapy in men with testicular germ cell cancer. Hum Reprod 2006;21:1781-6. 